\title{
VIOLÊNCIA CONTRA IDOSOS EM TRATAMENTO HEMODIÁLITICO
}

Fernanda Rosa de Oliveira Pires. Departamento de Enfermagem Universidade Federal de Sant Catarina; nandadode@hotmail.com;

Suzana Rosa; Departamento de Enfermagem da Universidade Federal de Santa Catarina (UFSC); suzana.srosa@hotmail.com;

Giordanna Nayara Chagas e Silva; Departamento de Enfermagem da Universidade Federal do Paraná (UFPR); giordanna@ufpr.br;

Susanne Elero Betiolli; Departamento de Enfermagem da Universidade Federal do Paraná (UFPR); susanne@ufpr.br;

Fernanda Cegan Gribner. Departamento de Enfermagem da Universidade Federal do Paraná (UFPR); fernandacegan@ufpr.br;

Bianca Dacoregio Martins; Departamento de Enfermagem da Universidade Federal de Santa Catarina (UFSC); biancaamd@outlook.com;

Karina Silveira de Almeida Hammerschmidt; Departamento de Enfermagem da Universidade Federal do Paraná (UFPR); ksalmeidah@ufpr.br;

\section{RESUMO}

Introdução: $\mathrm{O}$ aumento da população idosa gera preocupação mundial e de saúde pública a respeito da violência nesse público. Identificar os fatores e riscos que predispõe a violência contra 0 idoso é de extrema relevância para prevenção destas situações. Objetivo: Rastrear os riscos e fatores associados à violência contra idosos com doença renal crônica em tratamento hemodialítico. Método: Trata-se de estudo quantitativo, descritivo e exploratório realizado em duas unidades de tratamento hemodialítico da grande Florianópolis, com idosos em tratamento hemodialítico. A variável desfecho para risco de violência foi a escala Hwalek-Sengstock Elder Abuse Screening Test (H-S/EA). As variáveis independentes foram: idade, sexo, renda, estado civil, escolaridade, tempo em tratamento, número de pessoas que residem com o idoso, escala de Independência em Atividades da Vida Diária e escala de depressão geriátrica (GDS-15). Os dados foram analisados com a ferramenta Python, com operações de média aritmética simples, desvio padrão, cálculo de coeficientes de correlação, valor-p e cruzamento entre dados. Para cálculo de correlação foram utilizados os coeficientes de Person e Spearman e para análise de variância o método ANOVA. Resultados: Dos 43 idosos participantes, 29 $(67,44 \%)$ apresentaram risco de sofrer alguma forma de violência, com maior prevalência para o sexo masculino. Dentre os fatores independentes apenas os sintomas depressivos e renda apresentaram correlação positiva de significância estatística quando associados a riscos de violência entre idosos participantes. Conclusão: Os idosos apresentaram vulnerabilidade a risco de violência física psicológica, negligencia e financeira por terceiros, sendo maior parte destes do sexo masculino.

Palavras-chave: Idoso; Exposição à violência; Nefropatias; Diálise renal. 